\title{
How to Measure and Calculate Equivalent Series Resistance of Electric Double-Layer Capacitors
}

\author{
Rafael Vicentini ${ }^{1}$, Leonardo Morais Da Silva ${ }^{2, *} \mathbb{E}$, Edson Pedro Cecilio Junior ${ }^{1}$, \\ Thayane Almeida Alves ${ }^{1}$, Willian Gonçalves Nunes ${ }^{1}$ and Hudson Zanin ${ }^{1, *}$ \\ 1 Advanced Energy Storage Division, Center for Innovation on New Energies, Carbon Sci-Tech Labs, School of \\ Electrical and Computer Engineering, University of Campinas, Av Albert Einstein 400, Campinas, \\ SP 13083-852, Brazil; rafael.vicentini22@gmail.com (R.V.); edbig@uol.com.br (E.P.C.J.); \\ thayaneaalves@gmail.com (T.A.A.); nuneswillian40@gmail.com (W.G.N.) \\ 2 Department of Chemistry, Federal University of Jequitinhonha e Mucuri's Valley, Highway MGT 367, \\ km 583, 5000, Alto da Jacuba, Diamantina, MG 39100-000, Brazil \\ * Correspondence: lsilvamorais@hotmail.com (L.M.D.S.); hzanin@unicamp.br (H.Z.)
}

Received: 21 February 2019; Accepted: 28 March 2019; Published: 12 April 2019

check for updates

\begin{abstract}
Electric double-layer capacitors (EDLCs) are energy storage devices that have attracted attention from the scientific community due to their high specific power storage capabilities. The standard method for determining the maximum power $\left(P_{\max }\right)$ of these devices uses the relation $P_{\max }=U^{2} / 4 R_{\mathrm{ESR}}$, where $U$ stands for the cell voltage and $R_{\mathrm{ESR}}$ for the equivalent series resistance. Despite the relevance of $R_{\mathrm{ESR}}$, one can observe a lack of consensus in the literature regarding the determination of this parameter from the galvanostatic charge-discharge findings. In addition, a literature survey revealed that roughly half of the scientific papers have calculated the $R_{\mathrm{ESR}}$ values using the electrochemical impedance spectroscopy (EIS) technique, while the other half used the galvanostatic charge discharge (GCD) method. $R_{\mathrm{ESR}}$ values extracted from EIS at high frequencies $(>10 \mathrm{kHz})$ do not depend on the particular equivalent circuit model. However, the conventional GCD method better resembles the real situation of the device operation, and thus its use is of paramount importance for practical purposes. In the latter case, the voltage drop $(\Delta U)$ verified at the charge-discharge transition for a given applied current $(I)$ is used in conjunction with Ohm's law to obtain the $R_{\mathrm{ESR}}$ (e.g., $R_{\mathrm{ESR}}=\Delta U / \Delta I$ ). However, several papers have caused a great confusion in the literature considering only applied current $(I)$. In order to shed light on this important subject, we report in this work a rational analysis regarding the GCD method in order to prove that to obtain reliable $R_{\mathrm{ESR}}$ values the voltage drop must be normalized by a factor of two (e.g., $R_{\mathrm{ESR}}=\Delta U / 2 I$ ).
\end{abstract}

Keywords: equivalent series resistance; galvanostatic charge-discharge method; impedance technique; voltage drop; simulation of equivalent circuit models

\section{Introduction}

From urban mobility to portable electronics, fast charging devices are in growing demand [1-3]. In this scenario, electric double-layer capacitors (EDLCs), also known as supercapacitors, are very attractive energy storage devices with ultra-fast and short-term features [2-4]. To scale the characteristics of different EDLCs, the specific energy and power are commonly evaluated for practical purposes. The key parameters for the energy storage devices are regarded to the energy (Wh kg-1) and power $\left(\mathrm{W} \mathrm{kg}^{-1}\right)$ normalized per weight of the device (or the electrode material). In this sense, the maximum output energy $\left(E_{\max }\right)$ and power $\left(P_{\max }\right)$ are determined using the relations $E_{\max }=C U^{2} / 2$ and $P_{\max }$ $=U^{2} / 4 R_{\mathrm{ESR}}$, respectively, where $U$ stands for cell voltage, $C$ for specific capacitance, and $R_{\mathrm{ESR}}$ for equivalent series resistance (ESR) [5]. 
It is commonly verified in the literature that ESR can be properly determined using the electrochemical impedance spectroscopy (EIS) and the galvanostatic charge-discharge methods. In the case of EIS, the ESR value can be accurately determined at high frequencies $(>10 \mathrm{kHz})[6-8]$. This method is quite simple to be applied since it does not rely on the use of a particular equivalent circuit model for the device [9]. Despite the advantages offered by the EIS method, we must consider that the conventional GCD method better resembles the real operation conditions for supercapacitors, i.e., the use of this method in the laboratory studies is of paramount importance in order to obtain a more realistic experimental scenario resembling a real application.

In the case of the GCD method, the voltage drop $(\Delta U)$ verified at the charge-discharge transition for a given applied current (I) must be used in conjunction with Ohm's law to obtain the $R_{\mathrm{ESR}}$ (e.g., $R_{\mathrm{ESR}}=\Delta U / \Delta I$ and not $R_{\mathrm{ESR}}=\Delta U / I$ ) [5,10-16]. However, the 'ad hoc' adoption in the literature of non-standard normalizing factors for $\Delta U$ have led to a great confusion when findings present in different papers obtained for supercapacitor devices were compared.

The objective of the present work is to present a theoretical analysis of the GCD method to demonstrate that correct determination of $R_{\mathrm{ESR}}$ values requires the voltage drop to be normalized by a factor of two (e.g., $R_{\mathrm{ESR}}=\Delta U / 2 I$ and not $R_{\mathrm{ESR}}=\Delta U / I$ ). Simulations using canonic circuit models were carried out to emphasize the theoretical aspects inherent to the present work. Furthermore, a comparison of the theoretical electrochemical behaviors of these circuits in the frequency domain is presented using the EIS method.

\section{Fundamentals of the EIS and GCD Methods}

Figure 1a,b shows the simulated Nyquist (e.g., complex-plane) plots and the corresponding galvanostatic charge-discharge plots obtained as a function of the $R_{\mathrm{ESR}}$. It is worth mentioning that the impedance response verified at very high frequencies does not depend on the particular equivalent circuit model used in the simulation process [7,8,17-22]. In addition, for practical purposes, since the EIS is a steady-state technique obeying the linear theory of systems, one has that highly accurate values of resistances and capacitances can be obtained for EDLCs using a low amplitude sinusoidal voltage (e.g., $\delta U=10 \mathrm{mV}$ (peak-to-peak)) and scanning the frequency for various orders of magnitude (e.g., $\Delta f=100 \mathrm{kHz}$ to $10 \mathrm{mHz}$ ).

From the above considerations, simulations were accomplished using a canonic equivalent circuit model representing the ideal electrochemical response of a symmetric coin cell device where the positive and negative electrodes are identical. As it can be seen in the inset of Figure 1, the equivalent series resistance $\left(R_{\mathrm{ESR}}\right)$ is connected to a branch containing a capacitor $\left(C_{\mathrm{EDL}}\right)$, representing the charge storage process on the electrical double-layer (EDL) formed at the electrode/electrolyte interface, which stands in parallel with a leakage resistance $\left(R_{\mathrm{L}}\right)$. Despite the use of a canonic model, it is important noting that the $R_{\mathrm{ESR}}$-value obtained at high-frequencies always appear as a generalized resistance connected in series with the other circuit elements of the particular circuit model [4].

The simulation presented in Figure 1a was accomplished for different $R_{\mathrm{ESR}}$-values and using an $R_{\mathrm{L}}$ (impedance to leak current)-value of $1 \mathrm{M} \Omega$. Obviously, an ideal EDLC device has a very high leakage resistance $\left(R_{\mathrm{L}} \rightarrow \infty\right)$ and the phenomenon of frequency dispersion is absent, i.e., the complex-plane plot is characterized by a perfect vertical line (see Figure 1a). Thus, the extrapolation of this line on the real axis $\left(Z_{\text {real }}\right)$ at very high frequencies (e.g., $\omega \rightarrow \infty$ ) yields the desired value of the $R_{\mathrm{ESR}}$. Therefore, in real systems the true value of $R_{\mathrm{ESR}}$ is commonly determined by extrapolation using a high-frequency value of $\approx 1.0 \mathrm{kHz}$. 

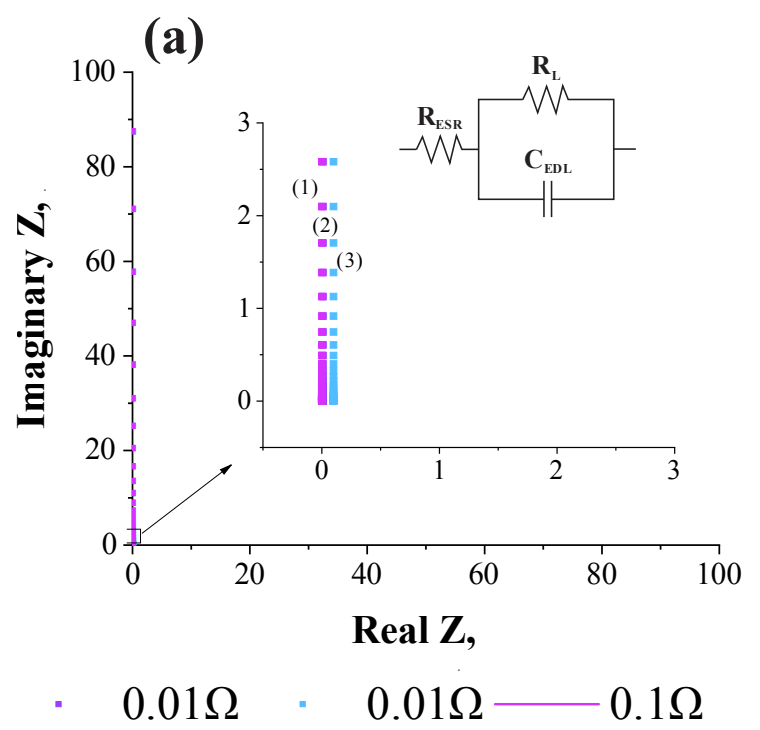

(b)

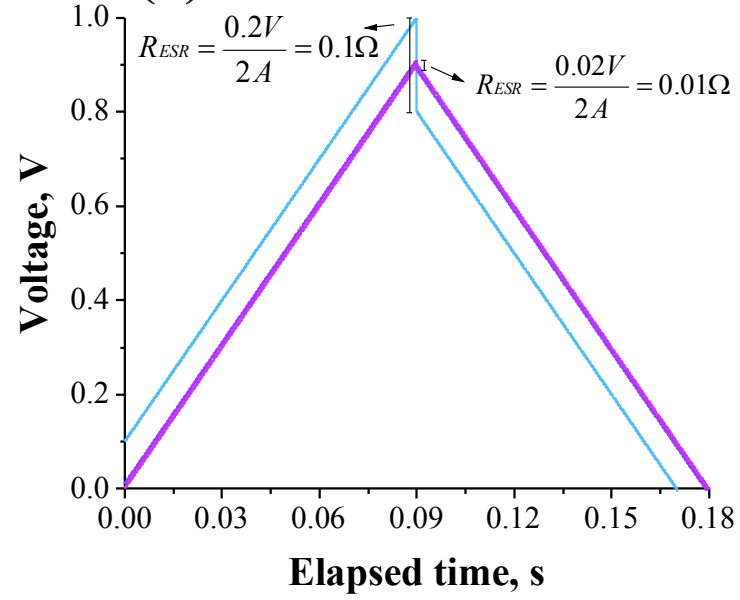

- $0.01 \Omega \quad 0.01 \Omega-0.1 \Omega$

Figure 1. Schematic representation of (a) the complex-plane plots and (b) the galvanostatic charge-discharge curves evidencing the voltage drop $\left(U_{\text {drop }}\right)$. The inset in Figure 1a shows the canonic circuit model. Simulation was carried out considering different values of the $R_{\mathrm{ESR}}$ and $R_{\mathrm{L}}=1 \mathrm{M} \Omega$.

Figure $1 \mathrm{~b}$ shows the galvanostatic charge-discharge curves evidencing the voltage drop $\left(U_{\text {drop }}\right)$ at the inversion of polarity. From the theoretical viewpoint, in this case the determination of the $R_{\mathrm{ESR}}$-value involves the application of a square wave current function with the inversion in polarity (e.g., $I_{(+)} \leftrightarrow I_{(-)}$and $\left.\left|I_{(+)}\right|=\left|I_{(-)}\right|\right)$.A voltage drop is observed at the reversal of polarization with a voltage increase after the sign of the current was reversed. During a continuous repetition of the charge-discharge processes, the positive (anode) and negative (cathode) electrodes were constantly charged and discharged, respectively, for equal times by applying positive $\left(I_{(+)}\right)$and negative $\left(I_{(-)}\right)$ currents of the same magnitude $\left(\left|I_{(+)}\right|=\left|I_{(-)}\right|\right)$. Therefore, for an ideal case where only a capacitive behavior exists $\left(R_{\mathrm{ESR}}=0\right)$ one would obtain as the response a symmetric triangular voltage wave since the capacitive voltage $\left(U_{\mathrm{c}}\right)$ increases linearly with the stored charge $(Q)$ for a given capacitance $(C)$, i.e., $\delta U_{\mathrm{c}}=\delta Q / C$. However, in real cases where $R_{\mathrm{ESR}}>0$ the anodic branch (e.g., the straight line with a positive slope) of the voltage wave referring to the charging process (e.g., $\left.\delta U_{\mathrm{c}(+)}=\delta Q_{(+)} / C\right)$ is displaced to more positive values by a constant value dictated by $U_{\mathrm{ESR}(+)}=R_{\mathrm{ESR}} \times I_{(+)}=$constant. Therefore, the instantaneous values of the overall voltage are given by $U_{\mathrm{i}}=U_{\mathrm{c}(+)}+U_{\mathrm{ESR}(+)}$. Accordingly, the cathodic branch (e.g., the straight line with a negative slope) of the voltage wave associated with the 
discharging process (e.g., $\delta U_{\mathrm{c}(-)}=\delta Q_{(-)} / C$ ) is displaced to more negative voltages due to the reversal in polarity of the applied current where $\delta I=I_{(+)}-I_{(-)}=2 I$, since $\left|I_{(+)}\right|=\left|I_{(-)}\right|$.

Intuitively, the instantaneous values of the overall voltage after the reversal in polarity is given by $U_{\mathrm{i}}=U_{\mathrm{c}}-U_{\mathrm{ESR}}=U_{\mathrm{c}}-R_{\mathrm{ESR}} \times 2 I$. In this sense, the overall voltage drop during reversal of the polarization is $U_{\mathrm{ESR}}=-R_{\mathrm{ESR}} \times 2 I$ since $\Delta U_{\mathrm{ESR}}<0$, i.e., $R_{\mathrm{ESR}}=\Delta U_{\mathrm{ESR}} / 2 I$ and, therefore, the voltage drop must be normalized by a factor of 2 [5].

\section{Theoretical Electric Response of the GCD Curves Using the Canonic Equivalent Circuit Model}

\subsection{Deriving the Theoretical Formula for the Equivalent Series Resistance}

To obtain the theoretical model for the calculation of the $R_{\mathrm{ESR}}$, the canonic circuit presented in Figure 2 representing the charge-discharge processes was employed to obtain the pertinent equations. In short, key equations for the charge and discharge processes and a combination of them are presented to obtain the desired theoretical model.
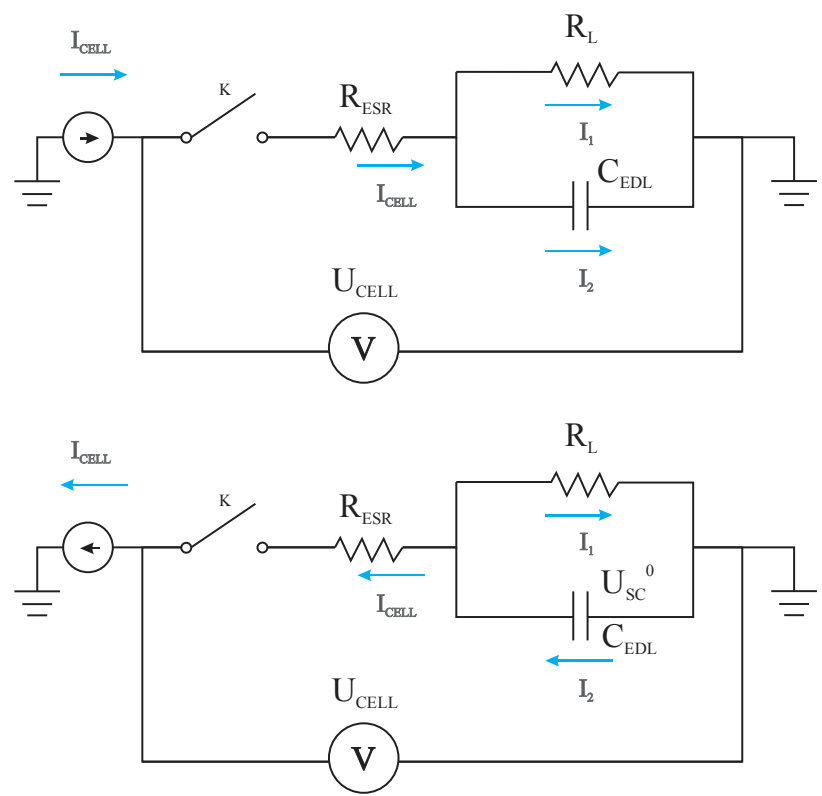

Figure 2. Electric circuits containing the canonic equivalent circuit model representing the electrochemical behavior of electric double layer capacitors during the charging and discharging processes carried out at constant current. Definitions: $I_{\text {cell }}$ is the constant current applied; $R_{\mathrm{ESR}}$ is the equivalent series resistance; $R_{\mathrm{L}}$ is the leakage resistance; $C_{\mathrm{EDL}}$ represents the equivalent capacitance of the symmetric coin cell; $U_{\text {cell }}$ is the overall cell voltage and $U_{\mathrm{SC}}{ }^{0}$ is the voltage across the $C_{\mathrm{EDL}}$ when the capacitor is fully charged.

According to the basic laws governing the theory of electric circuits the cell voltage during a charging process for the circuit presented in Figure 2 is given by the following relationship:

$$
U_{\text {cell }(\mathrm{t})}=R_{\mathrm{ESR}} I_{\text {cell }}+R_{L} I_{\text {cell }} \times\left[1-\exp \left(\frac{-t}{R_{L} C}\right)\right]
$$

In addition, it is possible to demonstrate that the function $U_{\text {cell }(t)}$ describing the transient response after the switcher $K$ is closed is given by the following equation:

$$
U_{\text {cell }(t)}=U_{\mathrm{sc}}^{0}-R_{\mathrm{ESR}} I_{\mathrm{cell}}-\left(U_{\mathrm{sc}}^{0}+R_{L} I_{\text {cell }}\right) \times\left[-1+\exp \left(\frac{-t}{R_{L} C}\right)\right]
$$

Using Equations (1) and (2), the theoretical galvanostatic charge-discharge curves accounting for the charging and discharging processes on $C_{\mathrm{EDL}}$, respectively, were obtained at constant current 
from numerical simulation (e.g., Simulink of PSIM software) using different $R_{\mathrm{ESR}}$-values (e.g., 0, 0.01, and $0.1 \Omega$ ) keeping the $R_{\mathrm{L}}=1.0 \mathrm{M} \Omega$ and $C_{\mathrm{EDL}}=0.1 \mathrm{~F}$ (see Figure 3). The anodic (positive) and cathodic (negative) currents were alternated between $+1 \mathrm{~A}$ and $-1 \mathrm{~A}$, respectively. In this case, a virtual controlled current source was applied referring to a charging time of $\sim 1 \mathrm{~s}$. Then, the current direction was reversed, thus characterizing the discharge of the $C_{\mathrm{EDL}}$. The magnitude of the charge and discharge currents was maintained so that the desired characteristics of the circuit could be graphically observed.

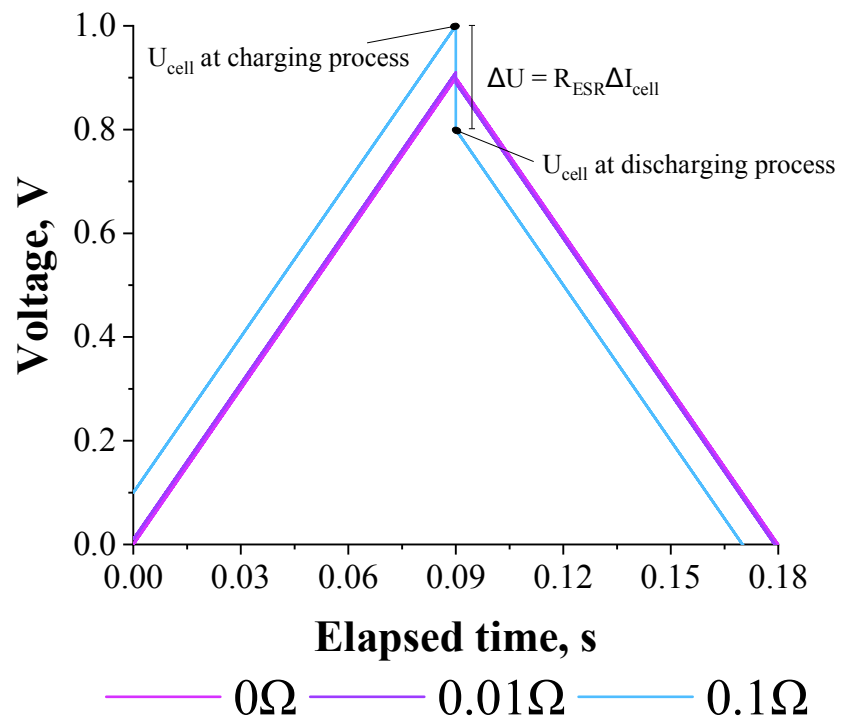

Figure 3. Simulation of the galvanostatic charge-discharge curves using different values of $R_{\mathrm{ESR}}$. Conditions: $R_{\mathrm{ESR}}=0,0.01$, and $0.1 \Omega ; R_{\mathrm{L}}=1.0 \mathrm{M} \Omega$; and $C_{\mathrm{EDL}}=0.1 \mathrm{~F}$. The anodic (positive) and cathodic (negative) currents were alternated between $+1 \mathrm{~A}$ and $-1 \mathrm{~A}$, respectively.

The $R_{\mathrm{ESR}}$-value can be determined by the voltage drop during the reversal of the polarity, i.e., when the charging process is discontinued to obtain the discharging curves. In the case of the cell voltage during the charging process (see Equation (1)), when $t \rightarrow \infty$ the capacitor is fully charged. Therefore, one has for this particular condition that:

$$
U_{\text {cell }(\mathrm{t})}=R_{E S R} I_{\text {cell }}+R_{L} I_{\text {cell }}
$$

Remembering that for the charging voltage when $t \rightarrow \infty$ one has that $R_{\mathrm{L}} I_{\text {cell }}$ is equal to the voltage in the capacitor $\left(U_{\mathrm{SC}}{ }^{0}\right)$, the following equation can be obtained:

$$
U_{\text {cell }(\mathrm{t})}=R_{E S R} I_{\text {cell (charging) }}+U_{\mathrm{sc}}^{0}
$$

In the case of the cell voltage for the discharging process $(t \rightarrow 0)$, when $t$ tends to zero the capacitor is fully discharged. Thus, for these conditions one has from Equation (2) that:

$$
U_{\text {cell }(\mathrm{t})}=U_{\mathrm{sc}}^{0}-R_{E S R} I_{\text {cell (discharging) }}
$$

Calculating the difference between Equations (1) and (2), the following relation is obtained:

$$
\Delta U=R_{E S R}\left(I_{\text {cell (charging) }}+I_{\text {cell(discharging })}\right)=2 R_{E S R}\left|I_{\text {cell }}\right|
$$

Since the charging and discharging current are equal in modulus. Finally, the theoretical expression for the equivalent series resistance is given by Equation (7):

$$
R_{E S R}=\frac{\Delta U}{2\left|I_{\text {cell }}\right|}
$$


Therefore, it become obvious from Equation (7) that the voltage drop must be normalized by a factor of 2 in order to obtain reliable findings during application of the GCD method. In a Supplementary Data we performed an alternative approach for same demonstration, please have a look.

\subsection{Validation of the Theoretical Expression Obtained for the Equivalent Series Resistance Using a Commercial} Supercapacitor of $200 \mathrm{~F}$

Figure 4 shows that the GCD curves obtained for a $200 \mathrm{~F}$ commercial supercapacitor (e.g., 2.7V D35H62 PTH S0016) as a function of the applied current (e.g., 1-2 A). For these high values of the applied currents the $\Delta U / \Delta I$ ratio was constant $(=25 \mathrm{~m} \Omega)$ [23].

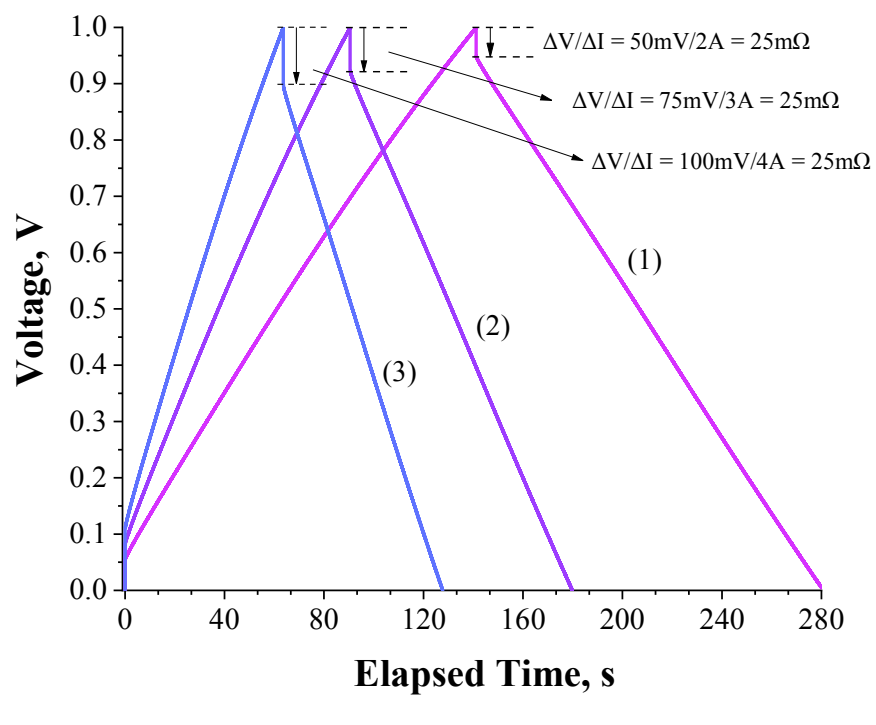

(1) $1 \mathrm{~A}-$ (2) $1.5 \mathrm{~A}-$ (3) $2 \mathrm{~A}$

Figure 4. Experimental GCD curves obtained for a 200F commercial supercapacitor (2.7V D35H62 PTH

S0016) as a function of the applied current.

Figure 5a shows the Nyquist plot obtained for the $200 \mathrm{~F}$ commercial supercapacitor (experimental conditions: $\delta U=10 \mathrm{mV}$ (peak-to-peak), $U_{\text {d.c. }}=0 \mathrm{~V}$, and $\Delta f=10 \mathrm{kHz}$ to $10 \mathrm{mHz}$ ). The impedance data of a real electric double-layer capacitor (EDLC) were quite different from the impedance response of a conventional (passive) capacitor due to the presence of the frequency dispersion phenomenon $[4,17]$. In fact, as discussed earlier by Conway [4], the EDLCs composed of high surface area porous carbon materials cannot be represented by a simple capacitance or even by a simple $R C$ circuit due to the influence of the ions in conjunction with the porous behavior exhibited by the electrode material, i.e., the high-frequency voltage hardly penetrates inside the narrow pores while the low-frequency voltage penetrates inside the porous electrode structure.

Figure 5a shows that at high frequencies the Nyquist plot was characterized by a semicircle which indicates the existence of a Faradaic leakage resistance in parallel with a capacitive element as a consequence of the contributions from the pseudocapacitance associated with surface redox functionalities present at the interfaces and/or edges of carbon particles [5]. Obviously, in practice, the $R_{\mathrm{ESR}}$-value is obtained at very high frequencies from extrapolation on the real axis of the Nyquist plot. Thus, it was verified in the present case that $R_{\mathrm{ESR}}=22 \mathrm{~m} \Omega$, which is in good agreement with that of $25 \mathrm{~m} \Omega$ obtained in this work using the classical GCD method.

At moderate frequencies, there was a linear region in the Nyquist plot with a phase angle of approximately $-45^{\circ}$, which indicates the presence of electrochemically active pores in the electrode structure. This characteristic behavior exhibited by porous electrodes was previously discussed in the literature [6]. On the contrary, the existence of a Warburg impedance $(W)$, which is also characterized by a phase angle of approximately $-45^{\circ}$, indicates that the electrochemical process is diffusion controlled 
during oxidation/reduction of the surface redox functionalities. In these different cases, the charge storage process is distributed over a network of $R$ and $C$ elements commonly represented by a semi-infinite transmission line of continuously connected $R$ and $C$ components. Despite the these considerations, one has in the special case of EDLCs characterized by a phase angle at high-frequencies of approximately $-45^{\circ}$ that is more plausible from the physicochemical viewpoint to consider the major influence of the porous electrode structure instead of a mass-transport controlled process since the real influence of the pseudocapacitance caused by the surface redox functionalities can be negligible in comparison with the purely electrostatic capacitance caused by the accumulation of the ions at the electrode/solution interface.

In addition, the analysis of the experimental findings obtained at low frequencies (see Figure 5a) revealed the presence of an inclined capacitive line. In fact, this behavior is predicted by the De Levie's model representing the electrochemical response of porous electrodes [7]. Considering the presence of non-idealities due to the frequency dispersion phenomenon, the inclined capacitive line can be represented by a $R^{*}-C P E$ circuit model where $Z_{\mathrm{CPE}}=1 / Y_{\mathrm{o}}(j \omega)^{n}$ and $n \cong 1$. In this case, $R^{*}$ contains information about the electrolyte resistance inside the pores while the parameter $Y_{\mathrm{o}}$ represents the non-ideal capacitance.

Figure $5 \mathrm{~b}$ shows the $R_{\mathrm{ESR}}$-values obtained from the GCD method as a function of the applied current. As it can be seen, a stationary value of $25 \mathrm{~m} \Omega$ was obtained for applied currents higher than $1 \mathrm{~A}$. These findings are in agreement with the experimental value of $22 \mathrm{~m} \Omega$ obtained using the EIS technique. It must be emphasized that for an applied current lower than $1 \mathrm{~A}$ the $R_{\mathrm{ESR}}$ values were overestimated in comparison with those obtained with the EIS technique. Therefore, the present study suggests that reliable values of the $R_{\mathrm{ESR}}$ must be obtained in the case of the GCD method using different currents in order to verify a stationary (constant) value. To the best of our knowledge, this important issue regarding the influence of the applied current on the $R_{\mathrm{ESR}}$ values has not been addressed in the literature.
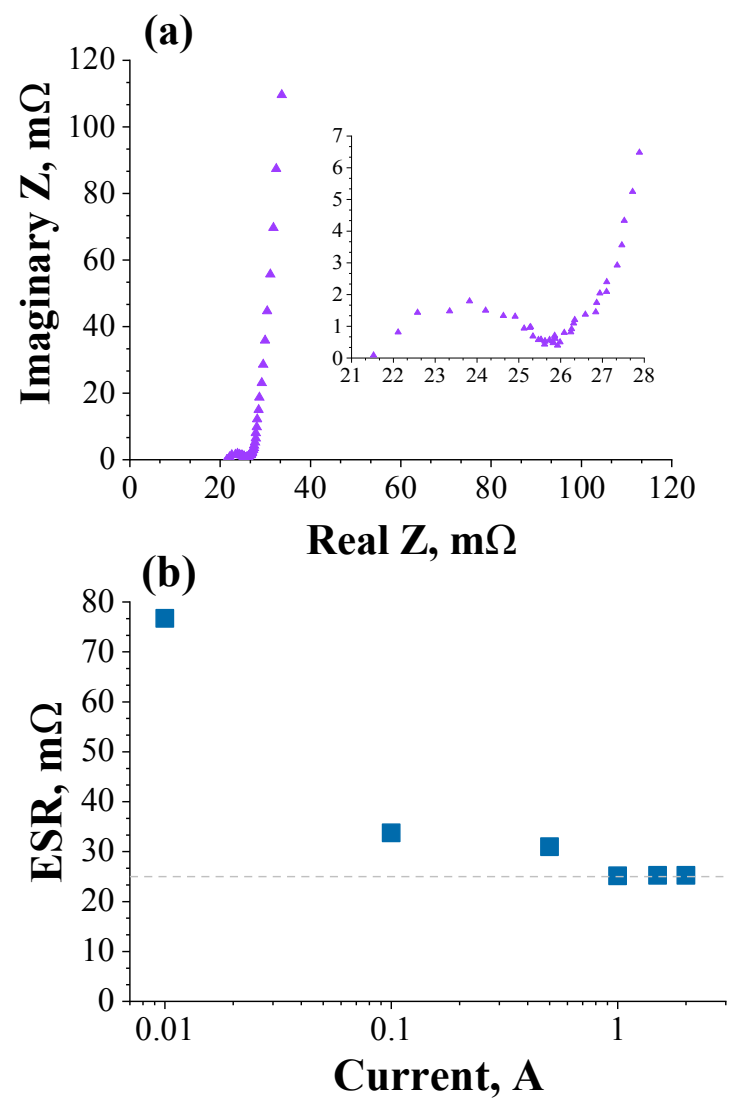

Figure 5. (a) Nyquist plot obtained for the commercial supercapacitor of 200 F. (b) Equivalent series resistance obtained from the GCD method as a function of the applied current. 


\section{Conclusions}

From the theoretical analysis of a canonic circuit model an Ohm-like equation was deduced for the determination of the equivalent series resistance $\left(R_{\mathrm{ESR}}\right)$ using the galvanostatic charge-discharge method. It was verified that the voltage drop must be normalized by a factor of two in order to obtain meaningful findings. The present work compared the applications of the electrochemical impedance spectroscopy (EIS) technique and galvanostatic charge/discharge (GCD) method in order to determine the value of the equivalent series resistance $\left(R_{\mathrm{ESR}}\right)$ present in electric double-layer capacitors (EDLCs). The derived equation was applied to obtain the $R_{\mathrm{ESR}}$ of a $200 \mathrm{~F}$ commercial supercapacitor.

In principle, the theoretical treatment presented in this work is strictly valid for EDLCs, i.e., where the battery-like Faradaic reactions are absent. Therefore, we may find discrepancies during the analysis of the experimental findings obtained for pseudocapacitors (PCs) where solid-state surface redox reactions occur. Further complications can also appear in the case of asymmetric capacitors since in these cases the positive and negative electrodes are not identical (i.e., they are composed of dissimilar materials).

Supplementary Materials: The following are available online.

Author Contributions: R.V. deduced the major equations, prepared all Figures, and experiments. E.P.C.J., W.G.N. and Leonardo confirm the theoretical analysis from R.V., L.M.D.S. helped in the development of the theoretical analysis. L.M.D.S. and H.Z. coordinate manuscript presentation and writing. T.A.A. prepares Figure 3 and simulation.

Funding: The authors are very grateful to financial support from the Brazilian funding agencies CNPq (301486/2016-6 DT-2 grant), FAPESP (2014/02163-7, 2017/011958-1), FAEPEX 2426/17, and CAPES (1740195). L.M. Da Silva wishes to thank the "Fundação ao Amparo à Pesquisa do Estado de Minas Gerais-FAPEMIG" (Project CEX-112-10), "Secretaria de Estado de Ciência, Tecnologia e Ensino Superior de Minas Gerais-SECTES/MG" (Support for the LMMA Laboratory), and "Conselho Nacional de Desenvolvimento Científico e Tecnológico-CNPq" (PQ-2 grant). The authors gratefully acknowledge support from Shell and the strategic importance of the support given by ANP (Brazil's National Oil, Natural Gas and Biofuels Agency) through the R\&D levy regulation.

Acknowledgments: We wish to thank student Lenon H. Costa for all discussions involving this topic.

Conflicts of Interest: The authors declare no conflict of interest.

\section{References}

1. Yu, A.; Chabot, V.; Zhang, J. Electrochemical Supercapacitors for Energy Storage and Delivery: Fundamentals and Applications, 1st ed.; CRC Press: Boca Raton, FL, USA, 2013.

2. Lu, M.; Beguin, F.; Frackowiak, E. Supercapacitors: Materials, Systems, and Applications, 1st ed.; Wiley-VCH: Weinheim, Germany, 2013.

3. Vicentini, R.; Costa, L.H.; Nunes, W.; Boas, O.V.; Soares, D.M.; Alves, T.A.; Real, C.; Bueno, C.; Peterlevitz, A.C.; Zanin, H. Direct growth of mesoporous Carbon on aluminum foil for supercapacitors devices. J. Mater. Sci. Mater. Electron. 2018, 29, 10573-10582. [CrossRef]

4. Conway, B.E. Electrochemical Supercapacitors: Scientific Fundamentals and Technological Applications; Plenum Press: New York, NY, USA, 1999.

5. Taberna, P.L.; Simon, P.; Fauvarque, J.F. Electrochemical Characteristics and Impedance Spectroscopy Studies of Carbon-Carbon Supercapacitors. J. Electrochem. Soc. 2003, 150, A292-A300. [CrossRef]

6. Taberna, P.L.; Portet, C.; Simon, P. Electrode surface treatment and electrochemical impedance spectroscopy study on carbon/carbon supercapacitors. Appl. Phys. A 2006, 82, 639-646. [CrossRef]

7. Basri, N.H.; Dolah, B.N.M. Physical and electrochemical properties of supercapacitor electrodes derived from carbon nanotube and biomass carbon. Int. J. Electrochem. Sci. 2013, 8, 257-273.

8. Niu, Z.; Zhou, W.; Chen, J.; Feng, G.; Li, H.; Ma, W.; Li, J.; Dong, H.; Ren, Y.; Zhao, D.; et al. Compact-designed supercapacitors using free-standing single-walled carbon nanotube films. Environ. Sci. 2011, 4, 1440-1446. [CrossRef]

9. Wang, Y.; Shi, Z.; Huang, Y.; Ma, Y.; Wang, C.; Chen, M.; Chen, Y. Supercapacitor Devices Based on Graphene Materials. J. Phys. Chem. C 2009, 113, 13103-13107. [CrossRef] 
10. Boukhalfa, S.; Evanoff, K.; Yushin, G. Atomic layer deposition of vanadium oxide on carbon nanotubes for high-power supercapacitor electrodes. Environ. Sci. 2012, 5, 6872-6879. [CrossRef]

11. Giorgi, A.; Mastragostino, M.; Soavi, F.; Di Fabio, A. Carbon-Poly(3-methylthiophene) Hybrid Supercapacitors. J. Electrochem. Soc. 2001, 148, A845-A850.

12. Caporali, S.; Soavi, F.; Balducci, A.; Bardi, U.; Mastragostino, M. Ionic liquids for hybrid supercapacitors. Electrochem. Commun. 2004, 6, 566-570.

13. Futaba, D.N.; Hata, K.; Yamada, T.; Hiraoka, T.; Hayamizu, Y.; Kakudate, Y.; Tanaike, O.; Hatori, H.; Yumura, M.; Iijima, S. Shape-engineerable and highly densely packed single-walled carbon nanotubes and their application as super-capacitor electrodes. Nat. Mater. 2006, 5, 987-994. [CrossRef] [PubMed]

14. Gualous, H.; Bouquain, D.; Berthon, A.; Kauffmann, J. Experimental study of supercapacitor serial resistance and capacitance variations with temperature. J. Source 2003, 123, 86-93. [CrossRef]

15. Keskinen, J.; Sivonen, E.; Jussila, S.; Bergelin, M.; Johansson, M.; Vaari, A.; Smolander, M. Printed supercapacitors on paperboard substrate. Electrochim. Acta 2012, 85, 302-306. [CrossRef]

16. Zhong, Y.; Zhang, J.; Li, G.; Liu, A. Research on Energy Efficiency of Supercapacitor Energy Storage System. In Proceedings of the 2006 International Conference on Power System Technology, Chongqing, China, 22-16 October 2006; IEEE: Chongqing, China, 2006; pp. 1-4.

17. Lasia, A. Electrochemical Impedance Spectroscopy and its Applications. Mod. Asp. Electrochem. 2002, 32, 143-248.

18. Liu, X.; Pickup, P.G. Ru oxide supercapacitors with high loadings and high power and energy densities. J. Source 2008, 176, 410-416. [CrossRef]

19. Shaijumon, M.M.; Ou, F.S.; Ci, L.; Ajayan, P.M. Synthesis of hybrid nanowire arrays and their application as high power supercapacitor electrodes. Chem. Commun. 2008, 2373-2375. [CrossRef] [PubMed]

20. Yan, J.; Wei, T.; Shao, B.; Ma, F.; Fan, Z.; Zhang, M.; Zheng, C.; Shang, Y.; Qian, W.; Wei, F. Electrochemical properties of graphene nanosheet/carbon black composites as electrodes for supercapacitors. Carbon 2010, 48, 1731-1737. [CrossRef]

21. Blomquist, N.; Wells, T.; Andres, B.; Bäckström, J.; Forsberg, S.; Olin, H. Metal-free supercapacitor with aqueous electrolyte and low-cost carbon materials. Sci. Rep. 2017, 7, 39836. [CrossRef] [PubMed]

22. Liu, C.; Yu, Z.; Neff, D.; Zhamu, A.; Jang, B.Z. Graphene-Based Supercapacitor with an Ultrahigh Energy Density. Nano Lett. 2010, 10, 4863-4868. [CrossRef] [PubMed]

23. Onsager, L. Deviations from Ohm's law in weak electrolytes. J. Chem. Phys. 1934, 2, 599-615. [CrossRef] 